\title{
Neuroimmune interactions and kidney disease
}

\author{
Sho Hasegawa ${ }^{1,2(\mathbb{D}}$, Tsuyoshi Inoue $^{2 \text { (i) }}$, Reiko Inagi ${ }^{2(\mathbb{D}}$ \\ 'Division of Nephrology and Endocrinology, University of Tokyo Graduate School of Medicine, Tokyo, Japan \\ ${ }^{2}$ Division of CKD Pathophysiology, University of Tokyo Graduate School of Medicine, Tokyo, Japan
}

\begin{abstract}
The autonomic nervous system plays critical roles in maintaining homeostasis in humans, directly regulating inflammation by altering the activity of the immune system. The cholinergic anti-inflammatory pathway is a wellstudied neuroimmune interaction involving the vagus nerve. CD4-positive T cells expressing $\beta 2$ adrenergic receptors and macrophages expressing the alpha 7 subunit of the nicotinic acetylcholine receptor in the spleen receive neurotransmitters such as norepinephrine and acetylcholine and are key mediators of the cholinergic antiinflammatory pathway. Recent studies have demonstrated that vagus nerve stimulation, ultrasound, and restraint stress elicit protective effects against renal ischemia-reperfusion injury. These protective effects are induced primarily via activation of the cholinergic anti-inflammatory pathway. In addition to these immunological roles, nervous systems are directly related to homeostasis of renal physiology. Whole-kidney three-dimensional visualization using the tissue clearing technique CUBIC (clear, unobstructed brain/body imaging cocktails and computational analysis) has illustrated that renal sympathetic nerves are primarily distributed around arteries in the kidneys and denervated after ischemia-reperfusion injury. In contrast, artificial renal sympathetic denervation has a protective effect against kidney disease progression in murine models. Further studies are needed to elucidate how neural networks are involved in progression of kidney disease.
\end{abstract}

Keywords: Autonomic nervous system, Cholinergic neurons, Imaging, three-dimensional, Optogenetics, Sympathetic nervous system, Vagus nerve stimulation

\section{Introduction}

The autonomic nervous system (sympathetic and parasympathetic systems) plays critical roles in maintaining homeostasis in humans [1]. Of note, close interactions

Received February 5, 2019; Revised May 28, 2019;

Accepted June 2, 2019

Edited by Gheun-Ho Kim, Hanyang University, Seoul, Korea

Correspondence: Tsuyoshi Inoue

Division of CKD Pathophysiology, University of Tokyo Graduate School of Medicine, 7-3-1 Hongo, Bunkyo-ku, Tokyo 113-8655, Japan. E-mail: tsinoue-tky@umin.ac.jp

Copyright (C) 2019 by The Korean Society of Nephrology

(a) This is an open-access article distributed under the terms of the Creative Commons Attribution Non-Commercial License (http://creativecommons. org/licenses/by-nc-nd/4.0/), which permits unrestricted non-commercial use, distribution, and reproduction in any medium, provided the original work is properly cited. between the nervous system and the immune system have recently been reported [2]. Some immune cells such as macrophages, dendritic cells, $\mathrm{T}$ cells, and B cells express receptors for neurotransmitters, directly receive information from the nervous system, and alter their activity in response to stimulation [3]. Recent advances have demonstrated that this neuroimmune interaction is closely associated with various disorders, including acute kidney injury (AKI) $[4,5]$, and may be a therapeutic target. This review summarizes recent insights into the molecular mechanisms of neuroimmune interactions and the association of this system with the pathophysiology of kidney disease.

\section{The cholinergic anti-inflammatory pathway}

Interaction between the nervous and immune systems 
was first reported in the 1980s. Sympathetic nerve fibers innervate several lymphoid organs, including the thymus, spleen, lymph nodes, gut-associated lymphoid tissue, and bone marrow, and are directed into zones of $\mathrm{T}$ lymphocytes and plasma cells rather than into nodular or B lymphocyte regions [6]. Moreover, pharmacological studies have demonstrated that $\beta 2$ adrenergic receptors are abundantly expressed on the cell membranes of immune cells [7]. These insights are indicative of an interaction between sympathetic neurons and the immune system. However, the detailed molecular mechanisms underlying such interactions are difficult to understand because the effects of sympathetic neurons on the immune system are complex and differ according to immune cell and neurotransmitter receptor types [8].

In contrast, the molecular mechanism underlying parasympathetic regulation of the immune system has been relatively clarified. This mechanism is termed the "in- flammatory reflex pathway" and is mediated by the vagus nerve. The peripheral vagus afferent pathway detects pathogen-associated molecular patterns, proinflammatory cytokines, immunoglobulins, and adenosine triphosphate and conveys signals to the nucleus tractus solitarius of the brainstem [9-16]. The efferent limb of the inflammatory reflex pathway is known as the cholinergic anti-inflammatory pathway (CAP) (Fig. 1). The vagus efferent pathway is composed of parasympathetic fibers, which innervate various internal organs and regulate their functions. In a lipopolysaccharide (LPS) model of inflammation, peripheral vagus nerve stimulation attenuates the release of tumor necrosis factor alpha (TNF- $\alpha$ ) [17], suggesting that the vagus nerve directly regulates the activity of the immune system. A series of studies by Tracey's group has demonstrated that macrophages expressing the alpha 7 subunit of the nicotinic acetylcholine receptor $(\alpha 7 \mathrm{nAChR})$ are the key mediators of the

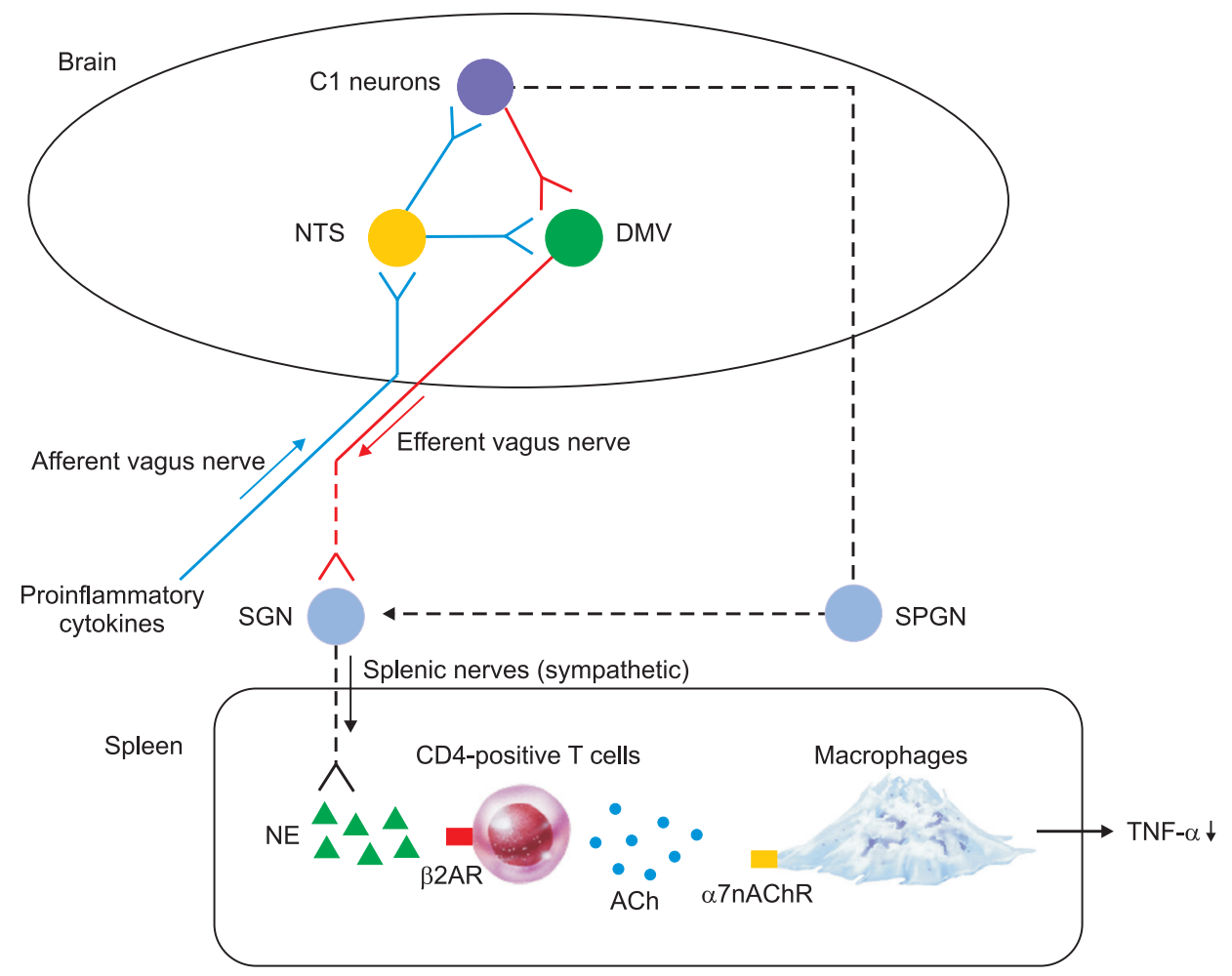

Figure 1. The cholinergic anti-inflammatory pathway. The cholinergic anti-inflammatory pathway bridges the nervous and immune systems. Afferent vagus nerves are stimulated by proinflammatory cytokines. The signal activates efferent vagus nerves via the nucleus tractus solitarius (NTS) and dorsal motor nucleus of the vagus (DMV) in the brain. Activated efferent vagus nerves stimulate splenic nerves, resulting in release of norepinephrine (NE). CD4-positive T cells in the spleen release acetylcholine (ACh) after NE binds to $\beta 2$ adrenergic receptors

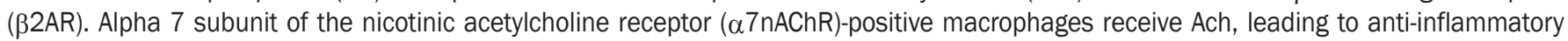
responses such as release of tumor necrosis factor alpha (TNF- $\alpha$ ). The dotted lines are strongly suggested, though not conclusively proven, pathways.

SGN, sympathetic ganglionic neuron; SPGN, sympathetic preganglionic neuron. 
interaction between the vagus nerve and the immune system. While $\alpha 7 \mathrm{nAChR}$ is predominantly expressed in neuronal tissues and plays an important role in generating action potentials, it is also expressed on macrophages and thereby inhibits nuclear translocation of nuclear factor kappa-light-chain-enhancer of activated B cells (NF$\kappa B)$ [18], activates the Janus kinase 2 (JAK2)-signal transducer and activator of transcription-3 (STAT3) pathway [19], and ultimately reduces the production of inflammatory cytokines. The inhibitory effect of vagus nerve stimulation on TNF- $\alpha$ release was abolished in systemic $\alpha 7$ nAChR knockout mice [20]. Moreover, acetylcholine and nicotine, which are agonists of nicotinic receptors, suppress TNF- $\alpha$ production in peritoneal macrophages induced by LPS but not in peritoneal macrophages derived from $\alpha 7 \mathrm{nAChR}$ knockout mice [20].

The spleen plays crucial roles in regulation of the CAP; the suppressive effects of vagus nerve stimulation on systemic TNF- $\alpha$ production are absent in mice that have undergone splenectomy [21]. Although the efferent pathway of the vagus nerve is predominantly composed of cholinergic fibers, the splenic nerve is exceptionally adrenergic, and no cholinergic fibers, which are the source of acetylcholine, exist in the spleen $[22,23]$. Thus, the source of acetylcholine in the spleen was unknown for many years. Rosas-Ballina et al [24] demonstrated that certain CD4-positive $\mathrm{T}$ cells mediate the interaction between

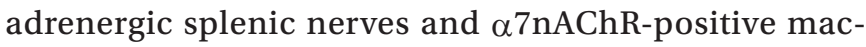
rophages via synthesis of acetylcholine in response to norepinephrine. The adrenergic terminals of the splenic nerve in the white pulp of the spleen come close to lymphocytes, including choline acetyltransferase (ChAT)expressing CD4-positive $\mathrm{T}$ cells, which primarily consist of memory T cells (CD44-high and CD62L-low) [24]. The anti-inflammatory effects of vagus nerve stimulation are absent in nude mice, which are devoid of functional $\mathrm{T}$ cells. In contrast, adoptive transfer of ChAT-expressing CD4-positive $\mathrm{T}$ cells into nude mice partially restores the effects of vagus nerve stimulation.

The importance of CD4-positive $\mathrm{T}$ cells in vagus nerve stimulation has been confirmed in several studies using a variety of methods. Vida et al [25] reported that vagus nerve stimulation fails to reduce serum TNF- $\alpha$ level in $\beta 2$ adrenergic receptor-deficient mice. Moreover, $\beta 2$ agonists inhibit cytokine production in the spleen and prevent systemic inflammation in wild-type mice but not in $\beta 2$ adrenergic receptor-deficient mice. Unlike typical regulatory $\mathrm{T}$ cells (CD25-positive), the transfer of CD4positive CD25-negative $\mathrm{T}$ cells to both $\beta 2$ adrenergic receptor-deficient mice and nude mice reestablishes the anti-inflammatory effects of vagus nerve stimulation.

In summary, CD4-positive T cells expressing $\beta 2$ adrenergic receptors in the spleen play crucial roles in the antiinflammatory effects of CAP by conveying splenic nerve activity to $\alpha 7 n A C h R-p o s i t i v e$ macrophages. The remaining question pertains to how sympathetic splenic nerves are activated in response to parasympathetic vagus nerve stimulation, which might include key evidence regarding the interaction of sympathetic and parasympathetic nerves and their regulation of the immune system.

\section{Vagus nerve stimulation in inflammatory disease}

The anti-inflammatory effects of vagus nerve stimulation are involved in many inflammatory diseases. Of note, vagus efferent fibers directly innervate the intestinal walls and regulate inflammation induced by resident macrophages independent of the spleen [26]. As a result, vagus nerve stimulation directly reduces inflammation of intestinal diseases such as colitis and ileus [27-29], which are conversely aggravated by vagotomy [30]. Although the vagus nerve does not directly innervate the joints, vagus nerve stimulation is now a therapeutic strategy for treatment of arthritis [31]. Vagus nerve stimulation suppresses limb inflammation (carrageenan-induced arthritis) in the acute setting [32], and chronic vagus nerve stimulation is reported to ameliorate collagen-induced arthritis [33,34]. Moreover, several reports have demonstrated that the anti-inflammatory effects of vagus nerve stimulation are observed in lifestyle diseases involving chronic inflammation such as diabetes [35] and hypertension [36].

\section{Vagus nerve stimulation and AKI}

AKI is a sudden loss of kidney function induced by various factors such as ischemia, sepsis, drugs, and toxins. Although the types of immune cells involved differ depending on the timing and causes of AKI, both the innate and adaptive immune systems play important roles in the pathophysiology of AKI [37-40]. One of the major causes of AKI is ischemia-reperfusion injury (IRI). Following renal IRI, neutrophils, natural killer (NK) cells, 
and NK T cells are recruited to the outer medullae of the kidneys and contribute to initiation of inflammatory cascades. Leukocyte infiltration is promoted by activation of the complement system and production of proinflammatory cytokines and chemokines [41] and is followed by monocyte infiltration. Monocytes differentiate into macrophages, which consist of various cell types with different functions. While pro-inflammatory (M1) macrophages promote tissue damage in the injury phase, antiinflammatory (M2) macrophages contribute to tissue repair [39,42].

Considering that the immune systems are closely related to the pathophysiology of AKI, involvement of neuroimmune interactions is an interesting notion. We previously demonstrated that vagus nerve stimulation is a potential therapeutic strategy for AKI [4]; vagus nerve stimulation 24 hours prior to IRI was successful in protecting the kidney from injury, preserving renal function and tissue morphology, and suppressing plasma TNF- $\alpha$ induction. This anti-inflammatory effect of vagus nerve stimulation was at least partly mediated by $\alpha 7 \mathrm{nAChR}-$ positive macrophages in the spleen. After lymphocytes harvested from the spleens of wild-type or $\alpha 7 \mathrm{nAChR}$ knockout donor mice treated with vagus nerve stimulation were transferred into wild-type recipient mice, the damage caused by renal IRI was significantly reduced in the recipient mice that received lymphocytes from wildtype donors. In contrast, no protection was observed in the recipient mice that received lymphocytes from $\alpha 7 n A C h R$ knockout donors [4]. This finding suggested

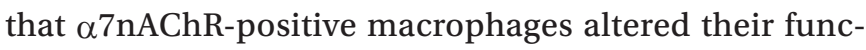
tion after vagus nerve stimulation and contributed to protection of the kidney against IRI. Although the number of macrophages in the kidney increased after IRI, the number of macrophages was comparable between wildtype and $\alpha 7 \mathrm{nAChR}$ knockout mice. However, the phenotype of the macrophages in the kidney differed between these mice. While Argl expression in the infiltrated macrophages in the kidney after IRI was suppressed after IRI, vagus nerve stimulation rescued Arg1 expression in wildtype mice but not in $\alpha 7 \mathrm{nAChR}$ knockout mice. Given that Arg1 is an M2 macrophage marker, the phenotypic change of the infiltrated macrophages toward M2 might be one mechanism underlying the anti-inflammatory effects of vagus nerve stimulation on IRI.

We further examined the role of $\alpha 7 \mathrm{nAChR}$-positive macrophages in the CAP [43]. While adoptive transfer of nicotine-treated peritoneal macrophages from wildtype mice offered protection against IRI, protection was not observed after transfer of nicotine-treated macrophages from $\alpha 7 \mathrm{nAChR}$ knockout mice, thereby indicating the importance of $\alpha 7 \mathrm{nAChR}$ in macrophages. Nicotineinduced genes that were more highly expressed in peritoneal macrophages from wild-type mice than in those from $\alpha 7 n A C h R$ knockout mice were identified by RNAsequencing analysis. As a result, hairy and enhancer of split-1 (Hes1), a basic helix-loop-helix transcription factor, was extracted. Vagus nerve stimulation induced Hesl expression in peritoneal macrophages. While Hes1 knockdown inhibited nicotine-induced TNF- $\alpha$ suppression, Hes1 overexpression suppressed LPS-stimulated TNF- $\alpha$ induction in macrophages and induced M2 macrophage markers. Moreover, adoptive transfer of Hesl-overexpressing macrophage cell lines suppressed the kidney damage induced by IRI. These experiments demonstrated that Hesl is a critical signaling molecule, through which $\alpha 7 \mathrm{nAChR}$-positive macrophages protect the kidney from injury after vagus nerve stimulation.

Although $\alpha 7 \mathrm{nAChR}$ macrophages in the CAP play important roles in the protective effects of vagus nerve stimulation, the full mechanism underlying vagus nerve stimulation-induced amelioration of kidney injury is not so simple. First, the roles of CD4-positive T cells expressing $\beta 2$ adrenergic receptors are not fully understood. In our previous study [43], the adoptive transfer of CD4positive splenocytes from vagus nerve stimulation-treated donor mice showed protective effects against IRI in recipient mice. Furthermore, kidneys were also protected when $\beta 2$ agonist-treated CD4-positive splenocytes were transferred to naïve recipients 24 hours before IRI. These results suggest that activating $\beta 2$ adrenergic receptors expressed in CD4-positive splenocytes might be an essential step underlying the CAP-mediated protective effects of vagus nerve stimulation in IRI. However, it has not been fully elucidated how and where these CD4-positive splenocytes interact with other immune cells and alter the immunological response in IRI. Second, the changes in renal hemodynamic caused by vagus nerve stimulation are unknown. As the vagus nerve stimulation utilized in our previous studies reliably produced a small reduction in heart rate without affecting blood pressure [4,43], we conclude that its protective effects were mainly derived 
from immunological alterations. However, we cannot conclude at this stage whether subtle hemodynamic changes within the kidney also induce independent protective effects against IRI. Third, the mechanism of afferent vagal stimulation-induced protection has not been elucidated. Renal protection was observed not only by efferent vagus nerve stimulation, but also by afferent vagus nerve stimulation [4]. Although afferent vagal stimulation activated the efferent vagal pathway on the contralateral side, protective effects were observed after left afferent vagal stimulation with the right vagal nerve blocked with local anesthetic, which suggests that the mechanism of afferent vagal stimulation-induced amelioration of kidney injury might differ from that of efferent vagal stimulation. Further studies are needed to clarify the mechanism of afferent vagal stimulation-induced protection against kidney injury. Finally, the effective timing of vagus nerve stimulation has not been fully clarified. In the clinical setting, transplanted kidneys are at risk of IRI. Vagus nerve stimulation in brain dead donor rats improves renal function and histology in recipient rats not only in an acute allograft rejection model, but also in a chronic allograft nephropathy model, indicating the potential clinical application of this technique [44]. Although previous studies have demonstrated the protective effects of vagus nerve stimulation before IRI, clinicians are also interested in the effects of post-intervention because onset of AKI is typically sudden and cannot be predicted in the clinical setting. Thus, studies to determine whether vagus nerve stimulation after IRI can ameliorate kidney injury are warranted.

\section{Ultrasound activates the CAP}

While ultrasound is a noninvasive imaging procedure in the clinical setting, it is also a candidate technique for activation of the CAP. Gigliotti et al $[45,46]$ reported that prior ultrasound suppresses inflammation in renal IRI. Anesthetized mice were exposed to an ultrasound protocol 24 hours before IRI. These ultrasound-treated mice exhibited preserved kidney morphology and function compared to sham-treated mice. Ultrasound exposure reduced the levels of systemic and renal cytokines such as interleukin 6 and TNF- $\alpha$, prevented the accumulation of neutrophils and macrophages in the kidney, and attenuated renal injury. Interestingly, size and weight of the spleen were negatively correlated with plasma creatinine level after 24 hours of reperfusion, suggesting that the spleen is involved in ultrasound-mediated tissue protection. Indeed, splenectomized mice receiving ultrasound before IRI had higher plasma creatinine level than mice receiving the same treatment with intact spleen [45]. Sympathetic denervation in the spleen by direct injection of 6-hydroxydopamine 14 days before IRI also abolished the ultrasound-mediated anti-inflammatory effects, demonstrating that splenic nerves are essential [46].

To determine the type of immune cells involved in ultrasound-mediated protection, Ragl knockout mice lacking $\mathrm{T}$ and $\mathrm{B}$ lymphocytes were used. The anti-inflammatory effects of prior ultrasound treatment were not observed in Ragl knockout mice. However, reconstitution of Rag1 knockout mice with wild-type CD4-positive T cells 10 days before ultrasound treatment restored ultrasoundinduced renal protection against IRI. These findings indicate that both the spleen and CD4-positive $\mathrm{T}$ cells are essential to ultrasound-mediated protection in renal IRI models.

Ultrasound-induced protective effects are also mediated by $\alpha 7 \mathrm{nAChR}$ signaling. In $\alpha 7 \mathrm{nAChR}$ knockout mice, ultrasound treatment did not protect the kidney from IRI [45]. Thus, bone marrow chimeras with wild-type and $\alpha 7 n A C h R$ knockout mice were used to determine whether hematopoietic or parenchymal $\alpha 7 n A C h R s$ mediate the protective effect of ultrasound. Such effects of ultrasound were preserved only in mice with bone marrow cells from wild-type mice. The parenchymal genotype was not related to the effects. Taken together, these findings suggest that the spleen (splenic nerves), CD4-positive T cells, and $\alpha 7 n A C h R-p o s i t i v e ~ m a c r o p h a g e s$, all of which are closely related to CAP activation, play important roles in ultrasound-mediated anti-inflammatory effects.

\section{Optogenetic approaches to elucidate the detailed neuronal networks of the CAP}

Conventional methods for functional analysis of neurons mainly use direct stimulation via small electrodes, including vagus nerve stimulation [4]. These methods cannot provide temporal and spatial nervous stimulation, preventing researchers from understanding the precise mechanism of neuronal networks. Optogenetics is a novel technique that utilizes light to control the ac- 
Hasegawa, et al. Neuroimmune interactions and kidney disease

tivity of neurons that have been genetically modified to express photosensitive ion transporters [47]. The discovery of channelrhodopsin-1 (ChR1) and channelrhodopsin-2 (ChR2) was the first important step for developing this technology $[48,49]$. These unique light-gated proton channels were first discovered in the green alga Chlamydomonas reinhardtii. Upon application of blue light ( $450 \pm 25$ $\mathrm{nm}), \mathrm{ChR} 2$ opens and functions as a non-selective cation channel, resulting in activation of neurons. Researchers started to introduce ChR2 into mammalian neurons using genetic technologies such as lentiviral gene delivery and achieved temporally precise, noninvasive control of neuronal activity [50]. Deisseroth et al [51] coined the term "optogenetics" in 2006.

The discovery of halorhodopsin inspired further development of optogenetics [52]. When yellow light (560 \pm $27.5 \mathrm{~nm}$ ) is applied to neurons expressing halorhodopsin, the chloride pump is activated, resulting in hyperpolarization and inhibition of the neurons. These light-sensing ion channel proteins can be introduced into specific cell types using genetic technologies such as the Cre-loxP system. Therefore, optogenetics enables control of the activity of specific neurons, which cannot be achieved by conventional electrical stimulation. This technique was selected as the "Method of the Year" by the Nature Publishing Group in 2010 [53].

Optogenetics is a useful tool for clarifying the mechanism of the CAP. Vagal afferent sensory neurons comprise several subgroups with specific markers, and optogenetic stimulation of each subgroup changes the function of the lung, heart, and gastrointestinal tract in different ways. For example, selective activation of P2ryl-positive neurons in vagal afferent neurons leads to apnea, while activation of Npy2r-positive neurons results in rapid shallow breathing [54]. In the digestive system, Gpr65-positive neurons innervating the intestinal villi are involved in homeostatic responses to ingested nutrients, while Glplr-positive neurons sense the mechanical distention of the stomach and intestines [54]. As the vagus and splenic nerves in the CAP are also composed of several subgroups, time- and cell-type-specific activation of neurons by optogenetic methods might clarify the detailed roles of each subgroup and thereby elucidate the selective neural circuits of the CAP.

\section{$C_{1}$ neurons and restraint stress in the CAP}

C1 neurons reside in the medulla oblongata and innervate many regions of the brain, including the dorsal motor nucleus of the vagus nerve, the paraventricular nucleus of the hypothalamus, other brainstem regions, and sympathetic and parasympathetic peripheral preganglionic neurons [55]. C1 neurons are involved in a broad range of physiological activities, including reproductive function during chronic glucose deficit [56], hypothalamic-pituitary-adrenal-mediated stress responses [57], glucose homeostasis [58,59], thermogenesis [60], breathing [61], and blood pressure [62]. C1 neurons are also involved in acute stress responses such as pain, hypoxia, infection, inflammation, hemorrhage, and hypoglycemia and thereby help the organism survive physical injury and disorders [55]. $\mathrm{Cl}$ neurons are thought to be associated with the inflammatory reflex because circulating cytokines and LPS strongly activate $\mathrm{Cl}$ neurons [63].

Using optogenetic stimulation, Abe et al [5] concluded that $\mathrm{C} 1$ neurons mediate a restraint stress-induced antiinflammatory reflex, which ameliorates kidney injury. First, they demonstrated that a brief period of restraint stress significantly protected the kidney from IRI. Adoptive transfer of splenocytes from restraint stress-exposed donor mice also protected the recipient's kidney from IRI. Moreover, the protective effects of restraint stress were not observed in $\alpha 7 \mathrm{nAChR}$ knockout mice. These results suggest that the anti-inflammatory effects of restraint stress are mediated via CAP activation. Given the role of $\mathrm{Cl}$ neurons in stress-mediated responses, Abe et al [5] hypothesized that $\mathrm{Cl}$ neurons were involved in the anti-inflammatory effects of restraint stress. Indeed, optogenetic $\mathrm{Cl}$ neuron stimulation by blue lasers protected the kidneys from IRI. It was also found that the spleen, $\beta 2$-adrenergic receptors, and $\alpha 7 \mathrm{nAChRs}$ were necessary for the protective effect, which strongly suggested activation of the CAP. Moreover, the protective effect of restraint stress was lost when $\mathrm{Cl}$ neurons were selectively inhibited by the Gi-coupled designer receptors exclusively activated by a designer drug system or destroyed, thus demonstrating that restraint stress-induced anti-inflammatory effects were mediated by $\mathrm{Cl}$ neuron activation. Subdiaphragmatic vagotomy and corticosterone receptor blockade did not abolish renal protection, demonstrating that $\mathrm{Cl}$ neuron-mediated CAP activation does not occur 


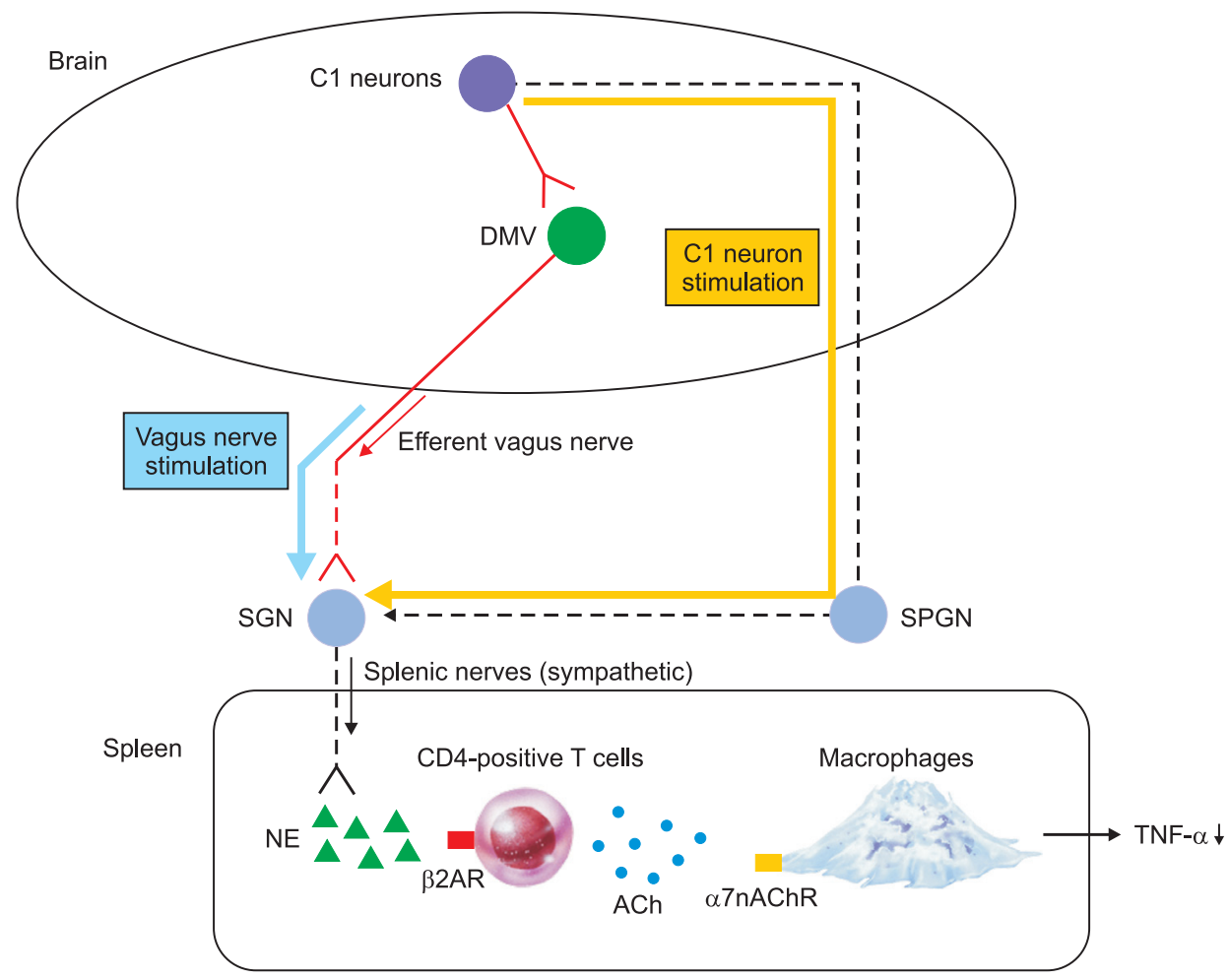

Figure 2. Mechanisms of cholinergic anti-inflammatory pathway activation between vagus nerve stimulation and C1 neuron stimulation. Subdiaphragmatic vagotomy does not abolish renal protection, indicating that C1 neuron-mediated cholinergic anti-inflammatory pathway (CAP) activation does not occur via a parasympathetic route but via a sympathetic route. The mechanism of CAP activation via C1 neurons (sympathetic) might differ from that via vagus nerve stimulation (parasympathetic). The dotted lines are strongly suggested, though not conclusively proven, pathways.

Ach, acetylcholine; $\alpha 7 \mathrm{nAChR}$, alpha 7 subunit of the nicotinic acetylcholine receptor; $\beta 2 \mathrm{AR}, \beta 2$ adrenergic receptor; DMV, dorsal motor nucleus of the vagus; NE, norepinephrine; SGN, sympathetic ganglionic neuron; SPGN, sympathetic preganglionic neuron; TNF- $\alpha$, tumor necrosis factor alpha.

via a parasympathetic route but via a sympathetic route (Fig. 2). These results suggest that the mechanism of CAP activation via $\mathrm{Cl}$ neurons (sympathetic) might differ from that via vagus nerve stimulation (parasympathetic). Further optogenetics studies are needed to fully understand the mechanism of CAP activation.

\section{Clinical application of vagus nerve stimulation}

As stated above, CAP activation by vagus nerve stimulation, ultrasound, and $\mathrm{C} 1$ neuron stimulation plays protective roles in murine renal IRI models. Among these procedures, vagus nerve stimulation is applied in the treatment and study of human diseases in the clinical setting. Vagus nerve stimulators are clinically used in the treatment of refractory epilepsy and medication-resistant depression [64]. Although transvenous vagus nerve stimulation failed to modulate the innate immune response during experimental endotoxemia in healthy volunteers [65], several studies have demonstrated that vagus nerve stimulators can relieve inflammation in human diseases such as rheumatoid arthritis and Crohn's disease [66,67]. After electrical stimulation of the vagus nerve, disease activity score improved significantly, systemic TNF- $\alpha$ level was reduced in rheumatoid arthritis patients, and withdrawal of treatment significantly worsened the severity of disease [66]. Vagus nerve stimulation also improved the biological parameters and endoscopic findings of patients with Crohn's disease over a six-month followup period [67]. Published representative clinical trials are listed in Table 1 [66-73]. Many clinical trials examining the effects of vagus nerve stimulation on a wide variety of disorders are in process.

In addition to implanted vagus nerve stimulators, noninvasive external devices have recently been developed [64]. A non-invasive transcutaneous device ameliorated 
Table 1. Published clinical trials on vagus nerve stimulation

\begin{tabular}{|c|c|c|c|}
\hline ClinicalTrials.gov identifier & Conditions & Subjects & Key findings \\
\hline NCT01792817 & $\mathrm{CH}$ & 133 & $\begin{array}{l}\text { The response rate was not significantly different (vs. sham) for the total population; } \\
\text { VNS provided significant benefits for episodic } \mathrm{CH} \text { but not for chronic } \mathrm{CH} \text {, which } \\
\text { affected the results in the total population [68]. }\end{array}$ \\
\hline NCT00305565 & TRD & 331 & $\begin{array}{l}\text { TRD patients who received VNS for } 22 \text { weeks showed significant improvement } \\
\text { compared with baseline. Higher electrical dose parameters were associated with } \\
\text { response durability [69]. }\end{array}$ \\
\hline NCT02367729 & $\begin{array}{l}\text { Functional disorder } \\
\text { of intestine }\end{array}$ & 115 & VNS has sustained efficacy for functional disorder of intestine in adolescents [70]. \\
\hline NCT00320372 & TRD & 795 & $\begin{array}{l}\text { The VNS group had better clinical outcomes than the control group, including a } \\
\text { significantly higher 5-year cumulative response rate and a significantly higher } \\
\text { remission rate [71]. }\end{array}$ \\
\hline NCT02686034 & Migraine & 248 & $\begin{array}{l}\text { The VNS group was superior to the sham group for pain freedom at } 30 \text { and } 60 \text { minutes } \\
\text { but not at } 120 \text { minutes after the first treated attack [72]. }\end{array}$ \\
\hline NCT01303718 & HF & 707 & VNS does not reduce the rate of death or HF events in chronic HF patients [73]. \\
\hline NCT01552941 & RA & 17 & $\begin{array}{l}\text { (Pilot study) } \\
\text { VNS significantly inhibited TNF production for up to } 84 \text { days in RA patients. RA severity } \\
\text { also improved significantly [66]. }\end{array}$ \\
\hline NCT01569503 & Crohn's disease & 7 & $\begin{array}{l}\text { (Pilot study) } \\
\text { Among the seven patients, two were removed from the study at } 3 \text { months for clinical } \\
\text { worsening and five evolved toward clinical, biological, and endoscopic remission [67]. }\end{array}$ \\
\hline
\end{tabular}

Representative clinical trials that have been published are listed in this table. While vagus nerve stimulation (VNS) showed clinical efficacy in cluster headache (CH), depression, migraine, and functional disorder of the intestine, a clinical trial for heart failure (HF) patients resulted in failure. Pilot studies for inflammatory diseases such as rheumatoid arthritis (RA) and Crohn's disease were also reported.

TRD, treatment-resistant depression.

acute ischemic injury in the rat brain [74] and downregulated the release of inflammatory cytokines in healthy humans [75]. As for kidney diseases, previous murine studies have reported anti-inflammatory effects of vagus nerve stimulation only when the procedure was performed before IRI. Thus, at this stage, clinical IRIs such as kidney transplantation and AKI after cardiac surgery are likely to benefit from vagus nerve stimulation, although no clinical data are available.

\section{Direct influence of renal sympathetic nerves on kidney diseases}

In this review, we mainly summarize how the autonomic nervous system regulates inflammation by altering immune activity. In addition to these immunological roles, nervous systems are directly related to the homeostasis of renal physiology. We recently visualized the threedimensional (3D) structure of sympathetic nerves and arteries in kidney tissue using the tissue clearing technique "CUBIC (clear, unobstructed brain/body imaging cocktails and computational analysis)" [76,77]. Whole- kidney 3D imaging revealed that sympathetic nerves are primarily distributed around arteries in the kidney (Fig. 3), suggesting that sympathetic innervation plays an important role in functional control of blood vessels [77]. Interestingly, time-course imaging data after IRI have demonstrated that innervation density is drastically reduced 4 days after surgery and gradually recovers somewhat by day 28 (Fig. 4). Moreover, norepinephrine level in kidney tissue (output of sympathetic nerves) is simultaneously reduced in injured kidneys [77]. These results illustrate sympathetic nervous abnormality during recovery from acute injury, although there remains a lack of clarity regarding the pathophysiological significance of sympathetic denervation after IRI.

Some previous reports suggest that artificial sympathetic denervation might exert protective effects in murine kidney disease models. Nagasu et al [78] demonstrated that artificial renal sympathetic denervation mitigates glomerular sclerosis in Dahl salt-sensitive hypertensive rats after unilateral nephrectomy by decreasing reactive oxygen species. Rafiq et al [79] have also shown that artificial renal sympathetic denervation suppresses de 

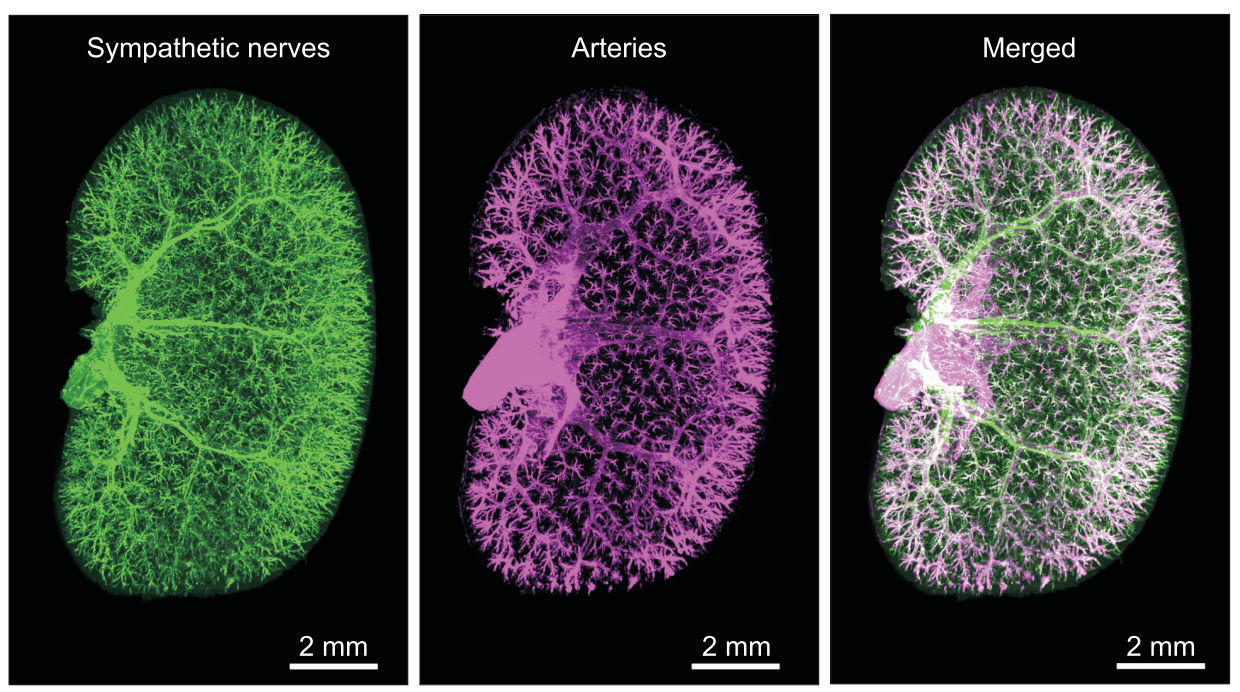

Figure 3. Three-dimensional distribution of sympathetic nerves and arteries in the kidney. The kidney was optically cleared and subjected to immunofluorescent staining with antibodies against tyrosine hydroxylase $(\mathrm{TH})$ and anti-alpha smooth muscle actin ( $\alpha \mathrm{SMA})$. Sympathetic nerves ( $\mathrm{TH}$, green) are primarily distributed in parallel with arteries ( $\alpha$ SMA, magenta).

Sham

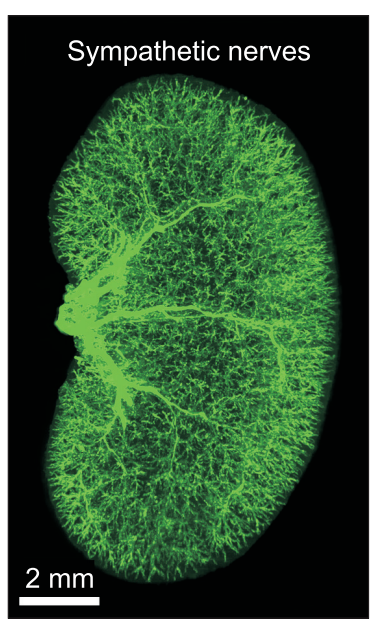

$\underline{\text { IRI (day 4) }}$

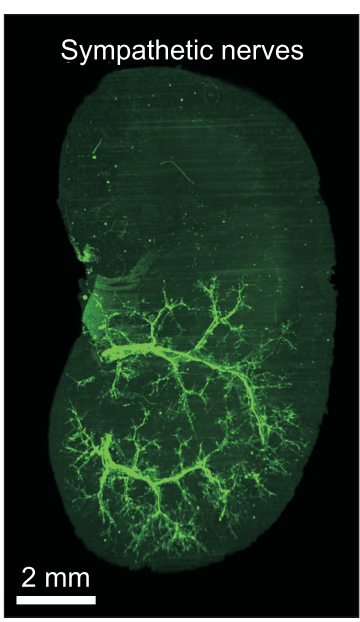

IRI (day 10)

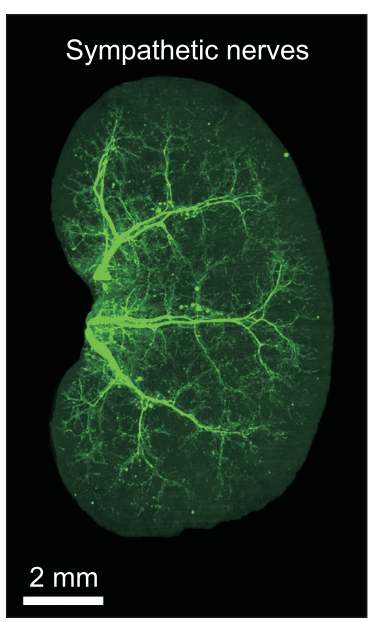

$\underline{I R I(d a y ~ 28)}$

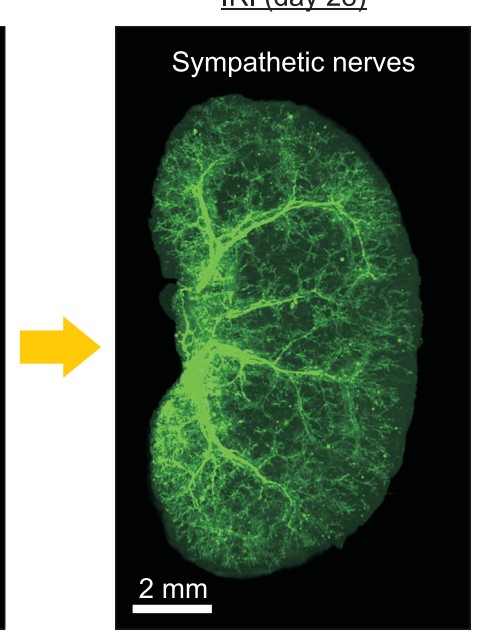

Figure 4. Time-course of sympathetic denervation after ischemia-reperfusion injury (IRI). Three-dimensional imaging reveals that sympathetic innervation density declines in injured kidneys. This sympathetic denervation was persistent even 28 days after injury, although innervation drastically decreased at day 4 and partially recovered over time.

novo podocyte injury and albuminuria in rats with aortic regurgitation. Furthermore, Kim and Padanilam [80] reported that renal denervation prevents fibrogenesis in mouse kidneys after IRI and ureteral obstruction [81]. Although these data strongly suggest that renal sympathetic activity might be involved in the pathophysiology of kidney diseases, the effects of denervation might be contextdependent, and further studies are needed to clarify the direct roles of sympathetic nervous activity in progression of kidney disease.

\section{Conclusions}

The autonomic nervous system directly regulates the immune system and plays important roles in various disorders. The key molecular mechanism underlying parasympathetic nerve regulation of the immune system has been partly clarified and is known as the CAP. Vagus nerve stimulation, ultrasound, and restraint stress activate the CAP and protect the kidneys from IRI. The novel approach of optogenetics has clarified that the antiinflammatory effects of restraint stress are mediated by $\mathrm{C} 1$ neurons. In addition to these immunological roles, nervous systems are directly related to homeostasis of 
renal physiology. Further studies are needed to elucidate how neural networks are involved in progression of kidney disease.

\section{Conflicts of interest}

Reiko Inagi has received research funding from KyowaHakko-Kirin. Sho Hasegawa and Tsuyoshi Inoue have no competing interests.

\section{Acknowledgments}

This work was supported by a Grant-in-Aid for the Japan Society for the Promotion of Science (JSPS) Research Fellowship (JSPS KAKENHI grant 19J11928 to Sho Hasegawa), Grant-in-Aid for Research Activity start-up (JSPS KAKENHI grant 18H06192 to Tsuyoshi Inoue), and Grant-in-Aid for Scientific Research (B) (JSPS KAKENHI grant $18 \mathrm{H} 02727$ to Reiko Inagi).

\section{Authors' contributions}

Sho Hasegawa wrote the original manuscript. Tsuyoshi Inoue and Reiko Inagi edited and revised the manuscript.

\section{References}

[1] Wehrwein EA, Orer HS, Barman SM. Overview of the anatomy, physiology, and pharmacology of the autonomic nervous system. Compr Physiol 2016;6:1239-1278.

[2] Pavlov VA, Tracey KJ. Neural regulation of immunity: molecular mechanisms and clinical translation. Nat Neurosci 2017;20:156-166.

[3] Kawashima K, Fujii T, Moriwaki Y, Misawa H. Critical roles of acetylcholine and the muscarinic and nicotinic acetylcholine receptors in the regulation of immune function. Life Sci 2012;91:1027-1032.

[4] Inoue T, Abe C, Sung SS, et al. Vagus nerve stimulation mediates protection from kidney ischemia-reperfusion injury through $\alpha 7 \mathrm{nAChR}+$ splenocytes. J Clin Invest 2016;126: 1939-1952.

[5] Abe C, Inoue T, Inglis MA, et al. C1 neurons mediate a stress-induced anti-inflammatory reflex in mice. Nat Neurosci 2017;20:700-707.

[6] Felten DL, Felten SY, Carlson SL, Olschowka JA, Livnat S. Noradrenergic and peptidergic innervation of lymphoid tissue. J Immunol 1985;135(2 Suppl):755S-765S.

[7] Brodde OE, Engel G, Hoyer D, Bock KD, Weber F. The betaadrenergic receptor in human lymphocytes: subclassification by the use of a new radio-ligand, (+/-)-125 Iodocyanopindolol. Life Sci 1981;29:2189-2198.

[8] Bellinger DL, Millar BA, Perez S, et al. Sympathetic modulation of immunity: relevance to disease. Cell Immunol 2008;252:27-56.

[9] Ek M, Kurosawa M, Lundeberg T, Ericsson A. Activation of vagal afferents after intravenous injection of interleukin1beta: role of endogenous prostaglandins. J Neurosci 1998; 18:9471-9479.

[10] Hermann GE, Rogers RC. TNFalpha: a trigger of autonomic dysfunction. Neuroscientist 2008;14:53-67.

[11] Page AJ, O'Donnell TA, Blackshaw LA. P2X purinoceptorinduced sensitization of ferret vagal mechanoreceptors in oesophageal inflammation. J Physiol 2000;523:403-411.

[12] Brouns I, Adriaensen D, Burnstock G, Timmermans JP. Intraepithelial vagal sensory nerve terminals in rat pulmonary neuroepithelial bodies express $\mathrm{P} 2 \mathrm{X}(3)$ receptors. Am J Respir Cell Mol Biol 2000;23:52-61.

[13] Niijima A, Hori T, Katafuchi T, Ichijo T. The effect of interleukin-1 beta on the efferent activity of the vagus nerve to the thymus. J Auton Nerv Syst 1995;54:137-144.

[14] Niijima A. The afferent discharges from sensors for interleukin 1 beta in the hepatoportal system in the anesthetized rat. J Auton Nerv Syst 1996;61:287-291.

[15] Steinberg BE, Silverman HA, Robbiati S, et al. Cytokinespecific neurograms in the sensory vagus nerve. Bioelectron Med 2016;3:7-17.

[16] Saeed RW, Varma S, Peng-Nemeroff T, et al. Cholinergic stimulation blocks endothelial cell activation and leukocyte recruitment during inflammation. J Exp Med 2005;201: 1113-1123.

[17] Borovikova LV, Ivanova S, Zhang M, et al. Vagus nerve stimulation attenuates the systemic inflammatory response to endotoxin. Nature 2000;405:458-462.

[18] Wang H, Liao H, Ochani M, et al. Cholinergic agonists inhibit HMGB1 release and improve survival in experimental sepsis. Nat Med 2004;10:1216-1221.

[19] de Jonge WJ, van der Zanden EP, The FO, et al. Stimulation of the vagus nerve attenuates macrophage activation by activating the Jak2-STAT3 signaling pathway. Nat Immunol 2005;6:844-851.

[20] Wang H, Yu M, Ochani M, et al. Nicotinic acetylcholine receptor alpha7 subunit is an essential regulator of inflam- 
mation. Nature 2003;421:384-388.

[21] Huston JM, Ochani M, Rosas-Ballina M, et al. Splenectomy inactivates the cholinergic antiinflammatory pathway during lethal endotoxemia and polymicrobial sepsis. J Exp Med 2006;203:1623-1628.

[22] Bellinger DL, Lorton D, Hamill RW, Felten SY, Felten DL. Acetylcholinesterase staining and choline acetyltransferase activity in the young adult rat spleen: lack of evidence for cholinergic innervation. Brain Behav Immun 1993;7:191204.

[23] Nance DM, Sanders VM. Autonomic innervation and regulation of the immune system (1987-2007). Brain Behav Immun 2007;21:736-745.

[24] Rosas-Ballina M, Olofsson PS, Ochani M, et al. Acetylcholine-synthesizing $\mathrm{T}$ cells relay neural signals in a vagus nerve circuit. Science 2011;334:98-101.

[25] Vida G, Peña G, Kanashiro A, et al. $\beta 2$-Adrenoreceptors of regulatory lymphocytes are essential for vagal neuromodulation of the innate immune system. FASEB J 2011;25:44764485.

[26] Matteoli G, Gomez-Pinilla PJ, Nemethova A, et al. A distinct vagal anti-inflammatory pathway modulates intestinal muscularis resident macrophages independent of the spleen. Gut 2014;63:938-948.

[27] Goverse G, Stakenborg M, Matteoli G. The intestinal cholinergic anti-inflammatory pathway. J Physiol 2016;594:57715780.

[28] Sun P, Zhou K, Wang S, et al. Involvement of MAPK/NF- $\mathrm{B}$ signaling in the activation of the cholinergic anti-inflammatory pathway in experimental colitis by chronic vagus nerve stimulation. PLoS One 2013;8:e69424.

[29] The FO, Boeckxstaens GE, Snoek SA, et al. Activation of the cholinergic anti-inflammatory pathway ameliorates postoperative ileus in mice. Gastroenterology 2007;133:12191228.

[30] Ghia JE, Blennerhassett P, Kumar-Ondiveeran H, Verdu EF, Collins SM. The vagus nerve: a tonic inhibitory influence associated with inflammatory bowel disease in a murine model. Gastroenterology 2006;131:1122-1130.

[31] McAllen RM, Cook AD, Khiew HW, Martelli D, Hamilton JA. The interface between cholinergic pathways and the immune system and its relevance to arthritis. Arthritis Res Ther 2015;17:87.

[32] Borovikova LV, Ivanova S, Nardi D, et al. Role of vagus nerve signaling in CNI-1493-mediated suppression of acute inflammation. Auton Neurosci 2000;85:141-147.
[33] Zhang P, Han D, Tang T, Zhang X, Dai K. Inhibition of the development of collagen-induced arthritis in Wistar rats through vagus nerve suspension: a 3-month observation. Inflamm Res 2008;57:322-328.

[34] Levine YA, Koopman FA, Faltys M, et al. Neurostimulation of the cholinergic anti-inflammatory pathway ameliorates disease in rat collagen-induced arthritis. PLoS One 2014;9: e104530.

[35] Wang L, Opland D, Tsai S, et al. Pten deletion in RIP-Cre neurons protects against type 2 diabetes by activating the anti-inflammatory reflex. Nat Med 2014;20:484-492.

[36] Carnevale D, Perrotta M, Pallante F, et al. A cholinergicsympathetic pathway primes immunity in hypertension and mediates brain-to-spleen communication. Nat Commun 2016;7:13035.

[37] Kinsey GR, Li L, Okusa MD. Inflammation in acute kidney injury. Nephron Exp Nephrol 2008;109:e102-e107.

[38] Rabb H, Griffin MD, McKay DB, et al. Inflammation in AKI: current understanding, key questions, and knowledge gaps. J Am Soc Nephrol 2016;27:371-379.

[39] Cao Q, Harris DC, Wang Y. Macrophages in kidney injury, inflammation, and fibrosis. Physiology (Bethesda) 2015;30: 183-194.

[40] Jang HR, Rabb H. Immune cells in experimental acute kidney injury. Nat Rev Nephrol 2015;11:88-101.

[41] Jang HR, Rabb H. The innate immune response in ischemic acute kidney injury. Clin Immunol 2009;130:41-50.

[42] Huen SC, Cantley LG. Macrophage-mediated injury and repair after ischemic kidney injury. Pediatr Nephrol 2015;30: 199-209.

[43] Inoue T, Abe C, Kohro T, et al. Non-canonical cholinergic anti-inflammatory pathway-mediated activation of peritoneal macrophages induces Hesl and blocks ischemia/reperfusion injury in the kidney. Kidney Int 2019;95:563-576.

[44] Hoeger S, Fontana J, Jarczyk J, et al. Vagal stimulation in brain dead donor rats decreases chronic allograft nephropathy in recipients. Nephrol Dial Transplant 2014;29:544549.

[45] Gigliotti JC, Huang L, Ye H, et al. Ultrasound prevents renal ischemia-reperfusion injury by stimulating the splenic cholinergic anti-inflammatory pathway. J Am Soc Nephrol 2013;24:1451-1460.

[46] Gigliotti JC, Huang L, Bajwa A, et al. Ultrasound modulates the splenic neuroimmune axis in attenuating AKI. JAm Soc Nephrol 2015;26:2470-2481.

[47] Montgomery KL, Iyer SM, Christensen AJ, Deisseroth K, 
Delp SL. Beyond the brain: optogenetic control in the spinal cord and peripheral nervous system. Sci Transl Med 2016;8:337rv5.

[48] Nagel G, Ollig D, Fuhrmann M, et al. Channelrhodopsin-1: a light-gated proton channel in green algae. Science 2002; 296:2395-2398.

[49] Nagel G, Szellas T, Huhn W, et al. Channelrhodopsin-2, a directly light-gated cation-selective membrane channel. Proc Natl Acad Sci U S A 2003;100:13940-13945.

[50] Boyden ES, Zhang F, Bamberg E, Nagel G, Deisseroth K. Millisecond-timescale, genetically targeted optical control of neural activity. Nat Neurosci 2005;8:1263-1268.

[51] Deisseroth K, Feng G, Majewska AK, Miesenböck G, Ting A, Schnitzer MJ. Next-generation optical technologies for illuminating genetically targeted brain circuits. J Neurosci 2006;26:10380-10386.

[52] Han X, Boyden ES. Multiple-color optical activation, silencing, and desynchronization of neural activity, with singlespike temporal resolution. PLoS One 2007;2:e299.

[53] Deisseroth K. Optogenetics. Nat Methods 2011;8:26-29.

[54] Chang RB, Strochlic DE, Williams EK, Umans BD, Liberles SD. Vagal sensory neuron subtypes that differentially control breathing. Cell 2015;161:622-633.

[55] Guyenet PG, Stornetta RL, Bochorishvili G, Depuy SD, Burke PG, Abbott SB. C1 neurons: the body's EMTs. Am J Physiol Regul Integr Comp Physiol 2013;305:R187-R204.

[56] I'Anson H, Sundling LA, Roland SM, Ritter S. Immunotoxic destruction of distinct catecholaminergic neuron populations disrupts the reproductive response to glucoprivation in female rats. Endocrinology 2003;144:4325-4331.

[57] Füzesi T, Wittmann G, Liposits Z, Lechan RM, Fekete C. Contribution of noradrenergic and adrenergic cell groups of the brainstem and agouti-related protein-synthesizing neurons of the arcuate nucleus to neuropeptide-y innervation of corticotropin-releasing hormone neurons in hypothalamic paraventricular nucleus of the rat. Endocrinology 2007;148:5442-5450.

[58] Verberne AJ, Sartor DM. Rostroventrolateral medullary neurons modulate glucose homeostasis in the rat. Am J Physiol Endocrinol Metab 2010;299:E802-E807.

[59] Zhao Z, Wang L, Gao W, et al. A central catecholaminergic circuit controls blood glucose levels during stress. Neuron 2017;95:138-152.

[60] Madden CJ, Tupone D, Cano G, Morrison SF. $\alpha 2$ Adrenergic receptor-mediated inhibition of thermogenesis. J Neurosci 2013;33:2017-2028.
[61] Burke PG, Abbott SB, Coates MB, Viar KE, Stornetta RL, Guyenet PG. Optogenetic stimulation of adrenergic C1 neurons causes sleep state-dependent cardiorespiratory stimulation and arousal with sighs in rats. Am J Respir Crit Care Med 2014;190:1301-1310.

[62] Wenker IC, Abe C, Viar KE, Stornetta DS, Stornetta RL, Guyenet PG. Blood pressure regulation by the rostral ventrolateral medulla in conscious rats: effects of hypoxia, hypercapnia, baroreceptor denervation, and anesthesia. $J$ Neurosci 2017;37:4565-4583.

[63] Li HY, Ericsson A, Sawchenko PE. Distinct mechanisms underlie activation of hypothalamic neurosecretory neurons and their medullary catecholaminergic afferents in categorically different stress paradigms. Proc Natl Acad Sci U S A 1996;93:2359-2364.

[64] Ben-Menachem E, Revesz D, Simon BJ, Silberstein S. Surgically implanted and non-invasive vagus nerve stimulation: a review of efficacy, safety and tolerability. Eur J Neurol 2015;22:1260-1268.

[65] Kox M, van Eijk LT, Verhaak T, et al. Transvenous vagus nerve stimulation does not modulate the innate immune response during experimental human endotoxemia: a randomized controlled study. Arthritis Res Ther 2015;17:150.

[66] Koopman FA, Chavan SS, Miljko S, et al. Vagus nerve stimulation inhibits cytokine production and attenuates disease severity in rheumatoid arthritis. Proc Natl Acad Sci U S A 2016;113:8284-8289.

[67] Bonaz B, Sinniger V, Hoffmann D, et al. Chronic vagus nerve stimulation in Crohn's disease: a 6-month follow-up pilot study. Neurogastroenterol Motil 2016;28:948-953.

[68] Silberstein SD, Mechtler LL, Kudrow DB, et al. Non-invasive vagus nerve stimulation for the acute treatment of cluster headache: findings from the randomized, double-blind, sham-controlled ACT1 study. Headache 2016;56:13171332.

[69] Aaronson ST, Carpenter LL, Conway CR, et al. Vagus nerve stimulation therapy randomized to different amounts of electrical charge for treatment-resistant depression: acute and chronic effects. Brain Stimul 2013;6:631-640.

[70] Kovacic K, Hainsworth K, Sood M, et al. Neurostimulation for abdominal pain-related functional gastrointestinal disorders in adolescents: a randomised, double-blind, shamcontrolled trial. Lancet Gastroenterol Hepatol 2017;2:727737.

[71] Aaronson ST, Sears P, Ruvuna F, et al. A 5-year observational study of patients with treatment-resistant depression 
treated with vagus nerve stimulation or treatment as usual: comparison of response, remission, and suicidality. Am J Psychiatry 2017;174:640-648.

[72] Tassorelli C, Grazzi L, de Tommaso M, et al. Noninvasive vagus nerve stimulation as acute therapy for migraine: the randomized PRESTO study. Neurology 2018;91:e364-e373.

[73] Gold MR, Van Veldhuisen DJ, Hauptman PJ, et al. Vagus nerve stimulation for the treatment of heart failure: the INOVATE-HF trial. J Am Coll Cardiol 2016;68:149-158.

[74] Ay I, Nasser R, Simon B, Ay H. Transcutaneous cervical vagus nerve stimulation ameliorates acute ischemic injury in rats. Brain Stimul 2016;9:166-173.

[75] Lerman I, Hauger R, Sorkin L, et al. Noninvasive transcutaneous vagus nerve stimulation decreases whole blood culture-derived cytokines and chemokines: a randomized, blinded, healthy control pilot trial. Neuromodulation 2016; 19:283-290.

[76] Susaki EA, Tainaka K, Perrin D, et al. Whole-brain imaging with single-cell resolution using chemical cocktails and computational analysis. Cell 2014;157:726-739.

[77] Hasegawa S, Susaki EA, Tanaka T, et al. Comprehensive three-dimensional analysis (CUBIC-kidney) visualizes abnormal renal sympathetic nerves after ischemia/reperfusion injury. Kidney Int 2019;96:129-138.

[78] Nagasu H, Satoh M, Kuwabara A, et al. Renal denervation reduces glomerular injury by suppressing NAD(P)H oxidase activity in Dahl salt-sensitive rats. Nephrol Dial Transplant 2010;25:2889-2898.

[79] Rafiq K, Noma T, Fujisawa Y, et al. Renal sympathetic denervation suppresses de novo podocyte injury and albuminuria in rats with aortic regurgitation. Circulation 2012; 125:1402-1413.

[80] Kim J, Padanilam BJ. Renal denervation prevents long-term sequelae of ischemic renal injury. Kidney Int 2015;87:350358.

[81] Kim J, Padanilam BJ. Renal nerves drive interstitial fibrogenesis in obstructive nephropathy. J Am Soc Nephrol 2013;24:229-242. 